\title{
Tile Size Selection of Affine Programs for GPGPUs using Polyhedral Cross-Compilation
}

\author{
Khaled Abdelaal and Martin Kong \\ The University of Oklahoma \\ Norman, Oklahoma, USA \\ \{khaled.abdelaal,mkong\}@ou.edu
}

\begin{abstract}
Loop tiling is a key high-level transformation which is known to maximize locality in loop intensive programs. It has been successfully applied to a number of applications including tensor contractions, iterative stencils and machine learning. This technique has also been extended to a wide variety of computational domains and architectures. The performance achieved with this critical transformation largely depends on a set of inputs given, the tile sizes, due to the complex trade-off between locality and parallelism. This problem is exacerbated in GPGPU architectures due to limited hardware resources such as the available shared-memory.

In this paper we present a new technique to compute resource conscious tile sizes for affine programs. We use Integer Linear Programming (ILP) constraints and objectives in a cross-compiler fashion to faithfully and effectively mimic the transformations applied in a polyhedral GPU compiler (PPCG). Our approach significantly reduces the need for experimental auto-tuning by generating only two tile size configurations that achieve strong out-of-the-box performance. We evaluate the effectiveness of our technique using the Polybench benchmark suite on two GPGPUs, an AMD Radeon VII and an NVIDIA Tesla V100, using OpenCL and CUDA programming models. Experimental validation reveals that our approach achieves nearly $75 \%$ of the best empirically found tile configuration across both architectures.
\end{abstract}

\section{CCS CONCEPTS}

- Software and its engineering $\rightarrow$ Compilers; $\bullet$ General and reference $\rightarrow$ Performance; $\bullet$ Mathematics of computing $\rightarrow \mathrm{Com}$ binatorial optimization; • Computer systems organization $\rightarrow$ Parallel architectures.

\section{KEYWORDS}

tile size selection, cross-compilation, polyhedral model, GPGPU, affine transformations, PPCG, CUDA, OpenCL.

ACM Reference Format:

Khaled Abdelaal and Martin Kong. 2021. Tile Size Selection of Affine Programs for GPGPUs using Polyhedral Cross-Compilation. In 2021 International Conference on Supercomputing (ICS '21), fune 14-17, 2021, Virtual

Permission to make digital or hard copies of all or part of this work for personal or classroom use is granted without fee provided that copies are not made or distributed for profit or commercial advantage and that copies bear this notice and the full citation on the first page. Copyrights for components of this work owned by others than ACM must be honored. Abstracting with credit is permitted. To copy otherwise, or republish, to post on servers or to redistribute to lists, requires prior specific permission and/or a fee. Request permissions from permissions@acm.org.

ICS '21, fune 14-17, 2021, Virtual Event, USA

(C) 2021 Association for Computing Machinery.

ACM ISBN 978-1-4503-8335-6/21/06.

https://doi.org/10.1145/3447818.3460369
Event, USA. ACM, New York, NY, USA, 14 pages. https://doi.org/10.1145/ 3447818.3460369

\section{INTRODUCTION}

Tiling is a critical loop transformation used to enhance a program's performance by improving its temporal locality [8, 27, 40, 48, 60]. This transformation has also been successfully adapted and applied to several computational domains, programming models and architectures. For instance, it has been widely used on iterative stencil computations [7, 20,37] and sparse matrix computations [25, 52].

In addition to rectangular tile shapes, a variety of other geometric forms trading communication and locality have also been explored, i.e. diamond tiles $[1,44]$, trapezoids $[16,17]$ and overlapped tiles $[49,67]$. Wonnacott and Strout [61] provide a summary of known techniques and comparisons. Tiling has also been combined with runtime abstractions [5, 33, 36, 53], extended to graphic processing units (GPUs) [2, 19, 24, 38, 59, 69, 70], and memory distributed computing $[12,13]$.

One particularity of this transformation is the need to provide parameters known as tile sizes. In fixed tiling schemes these values must be provided to the compiler in order to compute the high-order transformation. In contrast, a different class of tiling techniques known as parametric tiling $[3,26,28,31,55]$ can compute a single transformation for any valid tile size. In this class of schemes, tile sizes must be provided to enable the final code lowering. Several prior studies have shown the impact of selecting inadequate tile sizes. Thus, tile size selection has been the object of several studies $[11,65]$, quite often exploiting specific features of the underlying hardware, or combining it with the selection of unroll factors [32]. Unfortunately, a common limitation of these approaches is deciding the application of tile sizes in a separate tuning phase, which leads to ignoring the interplay among tile sizes, resource usage and the highlevel loop transformations being computed. In particular, expensive auto-tuning schemes that require exploring large candidate spaces with the corresponding experimental costs $[10,56,68]$ are common practice in high-performance computing (HPC).

In this paper we develop a novel approach to tile size selection for fixed rectangular tiling for GPGPUs. We propose a polyhedral crosscompilation technique, with PoCC [45] as the host compiler, and PPCG [59] as the target compiler. The goal of our cross-compilation strategy is to derive suitable tile size values that are later plugged into PPCG to produce at most two tiled variants for a GPU processor. Our approach builds on the single ILP scheduling approach $[47,57]$ to faithfully mimic the main transformations taking place in GPU-specific situations. Our approach starts by inserting loop dimensions to mimic the behavior of (to be) point loops. We then 
model the tileability of the program after applying this transformation pass. We leverage the legal space first proposed by Vasilache et al. using a $4 d+1$ schedule representation, and introducing constraints to drive the new schedule to find permutations wherein the tile and point dimensions form contiguous bands of loops. The selection of tile sizes are linked to two sets of balance constraints, one favoring tile sizes associated to innermost vector loops, and a second one favoring data reuse. We also model the usage of sharedmemory by defining memory quota constraints that become active depending on the loop fusion structure achieved as the result of the tiling transformation.

In summary, we make the following contributions:

- We devise, to the best of our knowledge, the first polyhedral cross-compilation technique based on the legal space formulation strategy where our goal is to compute tile sizes in a resource conscious fashion, and that attempt to maximize the shared-memory and L1-cache utilization;

- We introduce the notion of tile size balance to favor the expansion of tile size variables associated to schedule dimensions exhibiting either vector-parallelism or data reuse, while modeling the objectives in a $4 d+1$ schedule representation;

- We show that despite PoCC and PPCG being very different polyhedral compilers, we can model the core GPU transformations of PPCG in a faithful manner to effectively determine good sets of tile sizes;

- We conduct an extensive evaluation of our technique on two representative GPUs from different vendors and using different programming models. Our experiments demonstrate that, in average, we achieve nearly $75 \%$ of the best experimentally determined tile configuration after exploring large tile tuning spaces generated with PPCG.

The rest of this paper is organized as follows. Section 2 further motivates our research problem. Section 3 recaps background concepts on polyhedral compilation as well as the used terminology. Section 4 presents our formulation for selecting GPU-specific tile sizes that respect hardware constraints. Section 5 presents our results on two representative GPUs of different vendors. Section 6 discusses the related work on various (loop) tiling topics. Lastly, we present our conclusion in Section 7.

\section{MOTIVATION}

It is widely known that determining performance-optimal tile sizes is far from a trivial task. Tile sizes affect the performance of a computation in a number of ways. First, larger tile sizes increase the granularity of the computation. This can be beneficial for parallel innermost loops (usually good candidates for vectorization), but can also increase in excess the granularity of the computation, producing too few parallel iterations to distribute among the available compute units (cores, threads or streaming multi-processors). At the same time, larger tile sizes typically amount to better locality. However, too large tile sizes will also yield memory footprints that exceed cache capacities, inducing more cache misses. In contrast, small tile sizes favor parallelism exposure, but reduce the program's locality, possibly leading to the underutilization of memory resources. This problem becomes more complex as the effective tile memory footprint can vary, some times substantialy, with the

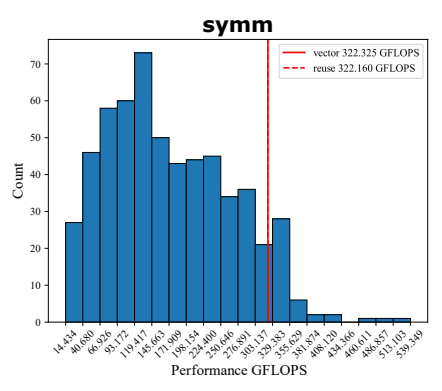

(a)

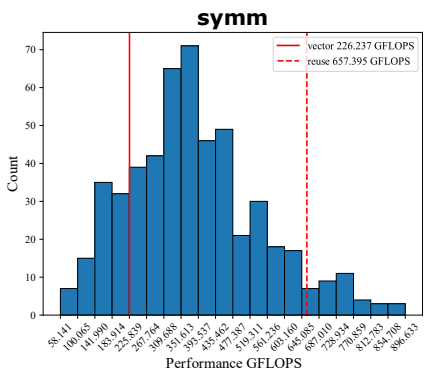

(b)
Figure 1: Performance histograms of Polybench symm benchmark (GFLOPS) when executed on (a) AMD Radeon VII GPU and (b) NVIDIA Tesla V100 GPU, for both the evaluated baseline configuration on PPCG and the configurations obtained with our selection scheme.

achieved loop fusion/distribution structure; Partially fused loops make ineffective usage of a cache, while perfectly fused loops can achieve full reuse on an array reference. In addition to these scenarios, caches with different associativity, being core private or shared, implicity or explicitly managed, and other hardware mechanisms such as memory prefetchers, make the problem of tile size selection extremely complex.

In the context of GPGPUs, where both software-managed and hardware-controlled caches are available, balancing the usage of these resources impose severe constraints on the set of legal tile sizes; This is owed to some tile size configurations exceeding the memory capacity, and producing failed kernel launches. To further motivate our problem, we show in Fig. 1 the performance distribution of the symm benchmark from the Polybench suite [46] on two representative GPUs, an NVIDIA Tesla V100 and an AMD Radeon VII (specifications can be found in Table 1). The evaluated space of tile sizes includes 525 different tile configurations on the NVIDIA V100 and 578 tile configurations on the AMD gpu; For the latter, a larger candidate space consisting of 799 variants was generated, yielding 221 failed configurations resulting from exceeding the shared-memory capacity. Each tile configuration was run 5 times; We report the mean value after excluding the fastest and slowest instances.

As one can observe, a very small fraction of the evaluated tiled space of configurations exhibits strong performance. On the NVIDIA GPU, less than $1.3 \%$ of the evaluated tile configurations fall within $1-10 \%$ of the fastest configuration; A similar trend can be observed on the AMD GPU, where about 10 instances yield a performance of 355 GFLOPS or above, representing less than $2 \%$ of the successfully explored space.

Hence, an efficient scheme is needed to find a high-performance tile size for each application without the need to exhaust a huge space. Our approach determines a high performance tile configuration using ILP constraints and objective functions. Our model takes resource information in consideration to decide two, nearoptimal, tile configurations for a given kernel and GPU target. Our approach vastly reduces the need to empirically explore large spaces of tile configurations. For example, on the Radeon VII, our approch 
achieves more than $2 \times$ of the median baseline performance, recording higher GFLOPS than $90 \%$ of the evaluated baseline tile configurations, as shown in Figrue 1a. Looking at Figure 1b, our approach achieves a performance higher than $93 \%$ of evaluated space, and around $1.8 \times$ better when compared to the median baseline performance on the NVIDIA GPU.

\section{BACKGROUND}

The polyhedral model excels at facilitating the computation and application of transformations on loop nests to exploit data locality and/or multi-level parallelism while ensuring the preservation of the program semantics. In particular, it's widely known for it's powerful composition of transformations [18], which are applied in a single pass via the polyhedral scanning process [4,23]. A polyhedral representation provides time and space insights of program transformations via a geometric view. We next review the main polyhedral abstractions and recap the formulation of the semantic preserving space of affine transformations [47, 57], which we use as basis for our cross-compilation tile size selection scheme.

\subsection{Static Control Parts (SCoPs)}

Affine programs are a sub-class of codes limited to compile-time analyzable code with static control-flow, and where bounds of loops and array index expressions are affine functions of the loop iterators surrounding the program statements. These conditions impose restrictions on the input code, resulting in excluding irregular codes such as tree-traversals [41, 54], array indirections and dynamic control conditions [58]. An example of a valid SCoP is shown in Figure 2, alongside with the graphical representation corresponding to the iteration domain of the single statement.

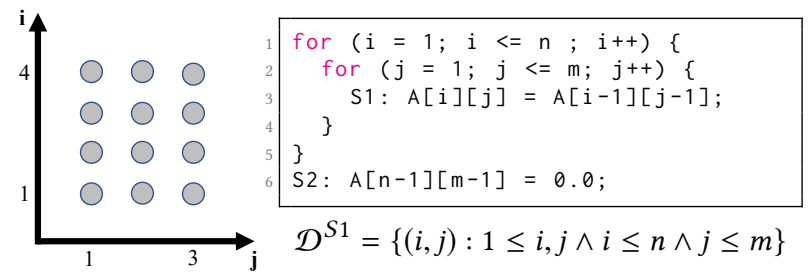

Figure 2: Code representing a valid SCoP (right) and the corresponding iteration domain figure for S1 (left) with $n=4$ and $m=3$

\subsection{Iteration Domains}

The restrictions previously recapped for affine codes with statically determinable control-flow allows to model the loop iterations of each program statement in a high-level, convex, closed form, using affine inequalities. The dynamic instances of each program statement are captured with a set $\mathcal{D}^{S} \in \mathbb{Z}^{d}$, where $d$ is the number of loops surrounding statement $S$. For example, in Figure 2, the iteration domain of statement $S 1$ is $\{(i, j): 1 \leq i \leq n \wedge 1 \leq j \leq m\}$.

\subsection{Access Relations}

Access functions/relations permit to map elements of the iteration domains to the memory locations accessed. Each reference $F^{A}$ of a multi-dimensional array $A$ is modeled using one or more affine functions with the iteration domain $\mathcal{D}^{S}$ as the domain of $F^{A}$, and the image of $F^{A}(\vec{i})$ being the memory elements accessed. As an illustration, we show the access functions for references $A[i-1][j-1]$ and $A[n-1][m-1]$ below:

$$
\begin{aligned}
F_{2}^{A} & =\left[\begin{array}{lllll}
1 & 0 & 0 & 0 & -1 \\
0 & 1 & 0 & 0 & -1
\end{array}\right]\left(\begin{array}{lllll}
i & j & n & m & 1
\end{array}\right)^{T}=\left[\begin{array}{l}
i-1 \\
j-1
\end{array}\right] \\
F_{3}^{A} & =\left[\begin{array}{lllll}
0 & 0 & 1 & 0 & -1 \\
0 & 0 & 0 & 1 & -1
\end{array}\right]\left(\begin{array}{lllll}
i & j & n & m & 1
\end{array}\right)^{T}=\left[\begin{array}{l}
n-1 \\
m-1
\end{array}\right]
\end{aligned}
$$

\subsection{Program Schedule}

The program schedule prescribes the order in which statement instances are executed. Schedules can be viewed as multi-dimensional time-stamps mapping each point in $\mathcal{D}^{S}$ to a logical execution date (which is enforced by the polyhedral code generator [4]). An example schedule representation is as follows: $\{S 1[i, j] \rightarrow[1, i, 0, j, 0]\}$ which is interpreted as statement instance $S 1(i, j)$ happening at the logical time $[1, i, 0, j, 0]$. More precisely, the schedule $\Theta^{S}$ of a statement $S$ is defined as a function of the iteration vector $\overrightarrow{i_{S}}$ and the vector of program parameters $\vec{p}$ (i.e. symbols $n$ and $m$ in the Fig. 2). Internally, the schedule can be modeled in matrix form, as per the example below:

$$
\Theta^{S}=\left(\begin{array}{ccccc}
\dot{\theta_{1,0}} & \dot{\theta_{1,1}} & \dot{\theta_{1,2}} & \dot{\theta_{1,3}} & \theta_{0,4} \\
\dot{\theta_{3,0}} & \dot{\theta_{3,1}} & \dot{\theta_{3,2}} & \dot{\theta_{3,3}} & \theta_{2,4} \\
\dot{\cdot} & \cdot & \cdot & \cdot & \theta_{4,4}
\end{array}\right)\left(\begin{array}{l}
i \\
j \\
n \\
m \\
1
\end{array}\right)=\left(\begin{array}{lllll}
0 & 0 & 0 & 0 & 1 \\
1 & 0 & 0 & 0 & 0 \\
0 & 0 & 0 & 0 & 0 \\
0 & 1 & 0 & 0 & 0 \\
0 & 0 & 0 & 0 & 0
\end{array}\right)=\left(\begin{array}{l}
1 \\
i \\
0 \\
j \\
0
\end{array}\right)
$$

Schedules can be lexicographically compared. For example, consider schedules $\Theta^{R}=\langle 0, i, 0, j\rangle$ and $\Theta^{T}=\langle 1, i, 0, j\rangle$, the latter being lexicographically greater than the former (i.e. all the instances of $\mathrm{T}$ will execute after all the instances of R); Similarly, a statement with the schedule $\Theta^{Q}=\langle 0, i, 1, j\rangle$ will have all its instances executing before those of $\mathrm{T}$, while being partially overlapping with statement $\mathrm{R}$ as a result of sharing the schedule prefix $\langle 0, i\rangle$ (leads to fusion on the outermost loop). Continuing with our example, the permutation of the loops surrounding $S 1$ can be achieved with the schedule $\{S 1[i, j] \rightarrow[j, i]\}$.

\subsection{Dependence Polyhedra}

A program dependence on an array reference $F^{A}$ between two statements $R$ and $S$, possibly being the same statement, is defined as the polyhedron:

$\mathcal{D}_{R, S}=\left\{\left\langle\overrightarrow{i_{R}}, \overrightarrow{i_{S}}\right\rangle: \overrightarrow{i_{R}} \in \mathcal{D}^{R} \wedge \overrightarrow{i_{S}} \in \mathcal{D}^{S} \wedge F^{A}\left(\overrightarrow{i_{S}}\right)=F^{A}\left(\overrightarrow{i_{R}}\right)\right\}$

The ordering constraints of a dependence are then encoded as legality constraints by introducing a semantic preserving condition $\Theta^{R} \prec \Theta^{S}$. Multiple dependence polyhedra embodying semantic ordering constraints, extracted via array data-flow analysis [14, 15], may be necessary to model each program dependence.

\subsection{Legal Space Constraints}

Next, we briefly recap the legal space formulation introduced by Vasilache [57] and later extended by Pouchet et al. [47]. In a nutshell, the formulation decomposes that satisfaction of each program dependence $\mathcal{D}_{R, S}$ into a predefined number of boolean variables 
$\delta^{\mathcal{D}_{R, S}}$, which is exactly the schedule depth. Dependences are weakly satisfied when a corresponding $\delta$ is zero, and strongly satisfied when their value is 1 . Moreover, the legality constraints recapped below are systematically nullified for all schedule levels deeper than the level at which a dependence is strongly satisfied. We also note that in this work we use a $4 d+1$ representation, where $d$ is the original loop-nest depth. The $4 d+1$ schedule format uses $2 d$ linear schedule dimensions and $2 d+1$ scalar rows. Moreover, the linear dimensions consist of $d$ interleaved tile and point dimensions. The legal space formulation vastly simplifies the selection of transformations. In particular, it favors the modeling of multiple objectives as well as the stirring of transformations to satisfy dependences at specific schedule levels.

$$
\begin{aligned}
& \forall \mathcal{D}_{R, S}, \forall l \in\{0 . .4 d\}, \delta_{l}^{\mathcal{D}_{R, S}} \in\{0,1\} \\
& \forall \mathcal{D}_{R, S}, \forall l \in\{0 . .4 d\}, \delta_{l}^{\mathcal{D}_{R, S}} \in\{0,1\}, \sum_{l=0}^{4 d} \delta_{l}^{\mathcal{D}_{R, S}}=1 \\
& \forall \mathcal{D}_{R, S}, \forall l \in\{0 . .4 d\}, \forall\left\langle\vec{i}_{R}, \vec{i}_{S}\right\rangle \in \mathcal{D}_{R, S}: \\
& \Theta_{l}^{S}\left(\vec{i}_{S}\right)-\Theta_{l}^{R}\left(\vec{i}_{R}\right) \geq-\sum_{c=0}^{l-1} \delta_{c}^{\mathcal{D}_{R, S}} \cdot(K \cdot \vec{n}+K)+\delta_{l}^{\mathcal{D}_{R, S}}
\end{aligned}
$$

\section{ESTIMATING TILE SIZES}

\subsection{Pre-Transformations and Schedule Format}

Pre-Tiling. The first step in our cross-compilation scheme is to pre-tile the input SCoP. Pre-tiling consists on introducing a new point loop $(P L)$ under each original loop. The $\mathrm{PL}$ is set to iterate $T_{i}$ times, where $T_{i}$ is a newly introduced program parameter. We introduce one such parameter for each lexically identifiable loop to guarantee that statements sharing the same loops will use the same tile sizes. Each PL is set to start at the coordinate of the immediately surrounding loop, which corresponds to a loop of the input SCoP. An example of the loop nest resulting from the pre-tiling step is shown next in Listing 1, where the loops with iterators $p i$ and $p j$ are the introduced point loops:

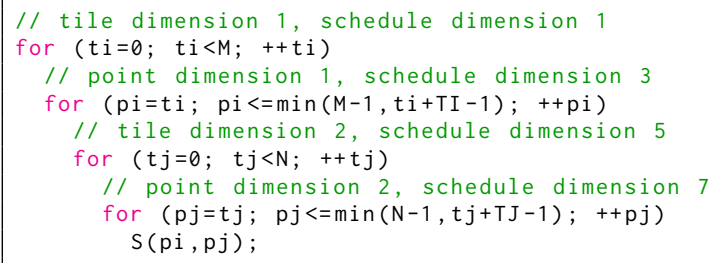

Listing 1: Example of the pre-tiling transformation from a 2D MxN loop

Henceforth, we assume that $d$ denotes the depth of the original (input) SCoP, i.e. prior to applying pre-tiling.

Dependence Selection. Once the pre-tiled SCoP is obtained, dataflow analysis [15] is performed to extract the dependence polyhedra. As the dependence structure in this $\mathrm{SCoP}$ is vastly different from the original one, we restrict the set of dependences to only those that also appear in the original SCoP, that match the source and target statements as well as their dependence types (flow, write, anti).
Second, we leverage the fact that our cross-compilation scheme allows us to take some liberties. Specifically, we perform a dependence filtering scheme where we only select at most one dependence between any pair of statements. The reason for this is to improve the scalability of our approach since the linearization step performed to build the legal space is known to be a compilation bottleneck. For convenience, we use $\delta_{c}^{R \rightarrow S}$ to denote each of the boolean dependence satisfaction variables for a given dependence polyhedron $\mathcal{D}_{R, S}$.

Schedule Format. After performing the pre-tiling step, we obtain a new schedule format, one which consists of $4 d+1$ schedule rows, $2 d+1$ of them being scalar dimensions representing the nesting structure of the produced loops (the even rows), and $2 d$ schedule rows associated to linear transformations (the odd rows). Specifically, any row $i$ s.t. $i \bmod 4=1$ corresponds to a loop in the input program, while if $i \bmod 4=3$, it corresponds to an inserted point loop. Below we show an example schedule in the $4 d+1$ format extracted from Listing 1 (after applying the pre-tiling step); The SCoP includes the original program parameters, $M$ and $N$, and the two newly introduced lexical tile sizes, $T I$ and $T J$. The example schedule also uses the column-vector $\beta$. We recall that the even entries of $\beta$ control and faithfully allow us to model the fused loop structure, while the odd components determine fine-grained loop fusion by means of constant loop shifting (also known as loop retiming) [18].

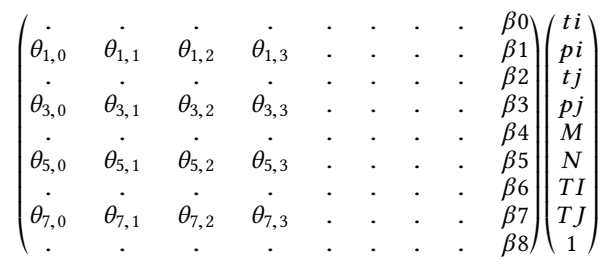

\subsection{Modeling Tileability}

To mimic a tiling scheme, even for an architecture such as a GPU, we introduce linear constraints to drive the selection of $\theta_{i, j}^{S}$ linear coefficients, i.e. where $i$ is odd. We introduce constraints for linear independence to guarantee that all loop dimensions are effectively used. We achieve this by introducing boolean shadow variables shadow $w_{i, j}^{S}$ one per $\theta_{i, j}^{S}, j<2 d$, building a column sum with them, and upper bounding the sum by $d$, the original loop depth. This objective is then lexicographically maximized. Each shadow boolean variable is connected to its corresponding schedule coefficient by an inequality of the form $\theta_{i, j}^{S} \leq K . \operatorname{shadow} w_{i, j}^{S}$, where $K$ is the fixed maximum value of any coefficient (NOTE: this is set to 40 in our scheduling approach). Thus, if some scheduling coefficient $\theta_{i, j}^{S}$ is used, the right-hand-side of the previous inequality will be lifted, making shadow $w_{i, j}^{S}=1$, otherwise it will be driven down towards zero.

In addition to the linear independence constraints, we also introduce an special class of permutation constraints to form the tile bands, i.e. sequences of linear dimensions (odd schedule rows) corresponding to the original tile and point loop dimensions. Intuitively, we seek to find a schedule that floats up all the original input loops while simultaneously sinking all the point loops introduced 
in the pre-tiling step. More concretely, the schedule that we seek to produce is one where: i) shadow $w_{i, j}^{S}=0$ if $j$ is odd and $i<2 d$; ii) $\operatorname{shadow}_{i, j}^{S}=1$ if $j$ is even and $i<2 d$. In a similar fashion, for the point dimensions we introduce constraints to favor the conditions: iii) shadow $w_{i, j}^{S}=0$ if $j$ is even and $i \geq 2 d$; iv) $\operatorname{shadow}_{i, j}^{S}=1$ if $j$ is odd and $i \geq 2 d$.

The potential of finding program schedules that exhibit the described conditions is maximized by linking together every pair of shadow variables corresponding to the same schedule row and input loop dimension to a boolean variable $\rho_{i, j}^{S}$ modeling each of the specific previous criteria. We recall that every original loop level $j$ has two schedule columns associated to it, with even columns referring to tile dimensions (after pre-tiling) and odd columns modeling introduced point loop dimensions. We also remark that the $\rho^{S}$ variables are created only for linear schedule dimensions (the odd rows of $\Theta^{S}$ ), as there is no need to model these conditions for scalar dimensions. More precisely, we use the below equalities to implement our main cost function:

$$
\begin{array}{r}
\operatorname{shadow}_{i, 2 j}^{S}-\operatorname{shadow}_{i, 2 j+1}^{S}=\rho_{i, j}^{S}, i<2 d \\
-\operatorname{shadow}_{i, 2 j}^{S}+\operatorname{shadow}_{i, 2 j+1}^{S}=\rho_{i, j}^{S}, \text { otherwise }
\end{array}
$$

The overall objective is then maximized level-by-level by summing the corresponding $\rho$ variables of each linear schedule dimension. Below we show the overall tiling objective, which in addition pushes the dependence satisfaction to all schedule rows not representing tile dimensions:

$$
O_{\text {tiling }}=\operatorname{Lexmax}\left\{\forall i \sum_{j} \rho_{i, j}^{S}, \sum_{i \notin t i l e \text { dim }} \delta_{i}^{\mathcal{D}_{R, S}}\right\}
$$

As an illustration, we show below a schedule meeting the previous requirements, which permutes the original loop order from $\langle t i, p i, t j, p j\rangle$ to $\langle t i, t j, p j, p i\rangle$.

$$
\Theta^{S}=\left(\begin{array}{ccccccccc}
\dot{1} & . & . & . & . & . & . & . & 1 \\
. & . & . & 0 & . & . & . & . & 0 \\
0 & 0 & 1 & 0 & . & . & . & . & 0 \\
. & . & . & . & . & . & . & . & 0 \\
0 & 0 & 0 & 1 & . & . & . & . & 2 \\
. & . & . & . & . & . & . & . & 0 \\
0 & 1 & 0 & 0 & . & . & . & . & 0 \\
. & . & . & . & . & . & . & . & 0 \\
p i \\
t j \\
p j \\
M \\
N \\
T I \\
T J \\
1
\end{array}\right)=\left(\begin{array}{c}
1 \\
t i \\
0 \\
t j \\
2 \\
p j \\
0 \\
p i \\
0
\end{array}\right)
$$

\subsection{Modeling Resource Constraints}

Modeling Available Memory. Given that the goal of our transformation is to derive a suitable set of tile size values for rectangular GPU tiling, several of the hardware constraints used in the following sections will be connected to the size of available sharedmemory. We thus assume that a memory size $M$ is given. $M$ is then converted to the number of scalar elements that would fit in it depending on the base data type size. So for instance, a memory of size $M=32 K B$ can fit 4096 double precision (DP) scalar elements. Later, we compute the logarithm of $M$ in a pre-defined tile base, obtaining mem $=\left\lfloor\log _{\text {tile_base }}(M)\right\rfloor$, and use this bound for several of the upcoming constraints.
Default Tile Size Bounds. We introduce one tile size variable $\tau_{i}^{S}$ for each loop dimension in the input SCoP. The bounds on the tile sizes are set between zero and $T_{\text {upper }}=\left\lfloor\log _{\text {tile base }}\left(R . P B S^{1 / R}\right)\right\rfloor$, where the tile base is set to $1.15, P B S$ is the problem size, and $R$ is the number of dimensions of a dominating array ${ }^{1}$. In the case of iterative stencils we use the problem size of the outermost space dimension. As an illustration, given a problem size of 1024 and a 2D dominating array, the upper bound produced would be $\log _{1.15}(64)$.

More on Tileability. Although we have taken the liberty to prune a subset of dependences obtained from the pre-tiled SCoP, it is nevertheless necessary to tie together the tile size variables to the dependence satisfaction variables, i.e. the $\delta^{S \rightarrow R}$ introduced for the legal space constraints (Sec.3.6). This requirement is not owed to legality, it is rather performance motivated, as faithfully modeling loop dimensions that are true candidates for tileability will allow a more accurate tile size selection. We recall that the boolean $\delta$ variables indicate a weak satisfaction (the dependence direction remains lexicographically non-negative up to the current schedule level) when they are zero, and a strong satisfaction when their value is 1 . Moreover, as a loop level is not permutable when it carries a dependence, we introduce the constraint $\tau_{i}^{S} \leq K .\left(1-\delta_{2 i+1}^{S \rightarrow R}\right)$, for all shared loop levels between statements $\mathrm{R}$ and $\mathrm{S}$, and $K$ being the fixed upper bound associated to the tile size variable. Then, whenever a linear schedule dimension $l$ carries a dependence, the upper bound on the corresponding tile size variable will be driven towards zero, disabling tiling at schedule level $l$.

Additional Tile Size Constraints and Objectives. The default tile size bounds are later extended with additional constraints. We introduce two sets of tile size constraints: i) memory footprint constraints, motivated and tied to hardware resources such as shared-memory; and ii) tile balance constraints, to keep a certain ratio among the chosen tile sizes. The purpose of the former type of constraints is to make the induced tile footprint to fit into the GPU shared-memory, whereas the latter kind permits to stir the selection of tile sizes to better match other hardware constraints (e.g. to exploit true vector units and SIMT parallelism). We note that this is, nonetheless, an optimistic approach, since the only way that we are influencing the scheduling decisions in the target compiler (PPCG in this case) is through the tile size values. To limit the memory footprint we create an optimization variable $m e m f^{S}$ for each statement $S$, which will aggregate the footprint of all its dominating arrays under the selected tile sizes. This objective is thus modeled as:

$$
m e m f^{S}=\sum_{\forall F \in S} \sum_{i=0}^{d-1} u s e d^{S, F, i} \cdot \tau_{i}^{S}
$$

where $u s e d^{S, F, i}$ is 0 if dimension $i$ is not present in the access function $F$ and 1 otherwise. Naturally, the footprint of a statement is the sum of all its dominating references.

The role of the balance constraints is to maintain a certain proportion among the tile size values. Intuitively, these constraints attempt to make the tile sizes proportional to a set of weights. We model two approaches for this, one where the tile size values (the

${ }^{1}$ An array reference $F$ is a dominating array if its dimensionality matches the maximum dimensionality of any array in the SCoP. 
$\tau_{i}^{S}$ variables) must be proportional to the frequency that each loop iterator appears on the fastest varying dimension (the rightmost dimension in row-major order storage), and a second approach where all tile sizes are inversely proportional to their frequency in the access functions of the statement's dominating arrays, but considering this time all loop dimensions. This translates to having larger tile sizes associated to loop dimensions that appear less in the access functions, i.e., data reuse. We thus call the former approach vector balance and the latter reuse balance.

Balancing Tile Sizes. To drive the selection of tile sizes we first compute the iterator frequencies on each dominating array, and aggregating them as needed depending on the balance approach taken. Moreover, we ignore constant and parametric shifts such as -1 in $A[i-1]$ and +2 in $B[2 k+2]$ when comparing arrays. For instance, for the main computing statement of the gemm kernel $(C[i][j]+=A[i][k] * B[k][j])$ the frequency of iterators would be 3, 3 and 2, for iterators $i, j$, k, respectively.

$$
O_{\text {balance }}: \forall S \operatorname{Lexmax} \sum_{i=0}^{d-1} B_{i}^{S} \cdot \tau_{i}^{S}
$$

The objective we use to achieve balance is shown in Eq.3. This is a per-statement objective, where $B_{i}^{S}$ is the weight associated to loop dimension $i$ and computed from the frequency of iterators appearing in the dominating array references. We remark that, as we make the distinction between vector balance and reuse balance, the former needs only to consider iterators present in the fastest varying dimension of the dominating arrays, whereas the latter considers all iterators in all dominating arrays. More precisely, for the reuse mode we define the weight associated to each tile size $\tau_{i}^{S}$ as

$$
B_{i}^{S}=\left\lceil\left(1+\sum_{\forall F, \forall k} u_{s e d} S, F, k\right) /\left(1+\sum_{\forall F} u_{s e d} S, F, i\right)\right]
$$

where $F$ is a dominating array reference. To complement the gemm example, the vector balance mode will aim to maximize the tile size associated to the original loop dimension $i$, whereas the reuse mode will prefer to maximize the tile size associated to the input dimension $\mathrm{k}$.

One last consideration we make is to relax the constraint on tile sizes by lifting the upper bound on each $\tau_{i}^{S}$ to

$$
T_{\text {upper }}+\left\lceil\log _{\text {tile_base }}\left(B_{i}^{S}\right)\right\rceil
$$

Naturally, Eq. 5 becomes the only effective fixed upper bound on $\tau_{i}^{S}$.

Modeling the Fusion Structure. Modeling the fusion/distribution loop structure that a GPU polyhedral compiler such as PPCG will produce is a critical step to properly select good tile sizes. The intuition here is that multiple GPU kernels will be created for a single SCoP. As a result, program statements mapped to different compute kernels can use a higher fraction of the compute resources, which translates to being able to select larger tile sizes.

Consequently, the approach we take to adjust the selection of tile sizes is to project and lift the maximum memory quota allocated to each program statement depending on one of three possible preidentified loop fusion scenarios. The three scenarios we consider are shown in Listing 2 - 4.

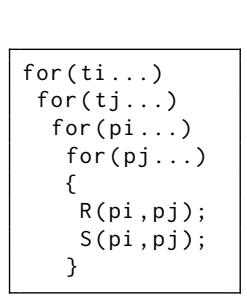

Listing 2: Full
Tile Fusion:
$\sigma_{1}=1 \wedge \sigma_{2}=0$

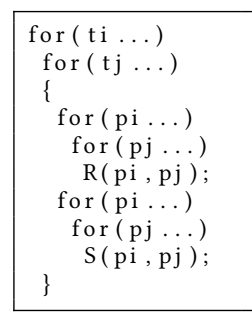

\section{Listing $\quad 3$ : \\ Partial}

Tile Fusion:

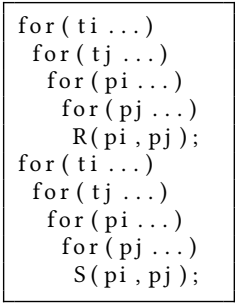

Listing 4: Full Tile Distribution: $\sigma_{1}=$ $0 \wedge \sigma_{2}=0 / 1$
The insight of this step is that when two or more statements are fully fused, and assuming there is data reuse among them (i.e. sharing an array reference such as $A[i][j])$, the tile memory footprint does not increase, and the corresponding tile sizes can grow towards their upper bound. This scenario is depicted in the leftmost code snippet of Listing 2. The second scenario pertains to partial tile fusion, wherein two or more statements share the same tile-band, but are distributed at the first point loop dimension, i.e. where the actual tile commences. The implication here is that statements fused under the same tile-band will have to compete for memory resources, resulting in a lower amount of data sharing, and which in turn will decrease the per-statement memory quota. The corresponding scenario is the code snippet in Listing 3. The third and final loop fusion scenario (Listing 4) considered is fully distributed loop nests. Such case will normally result from the need to obtain two outer parallel loop dimensions, which will induce loop distribution if the program dependences cannot be pushed inward. Correctly predicting this scenario is necessary since its occurrences also allow to increase the per-statement memory quota. Moreover, this case also implies that statements will be mapped to different compute kernels.

The discussed fusion scenarios are modeled using two boolean variables per statement, $\sigma_{1}^{S}$ and $\sigma_{2}^{S}$, which allow to shift the memory quota upper bound assigned to each dominating reference $F \in S$. Using these variables, the constraints for the full tile fusion scenario become effective when $\sigma_{1}^{S}=1$. Partial tile fusion is enabled when $\sigma_{1}^{S}=1$ and $\sigma_{2}^{S}=1$. We note that $\sigma_{2}^{S}$ can only be 1 when $\sigma_{1}^{S}$ is also 1 . The default case is full tile distribution, which becomes active when $\sigma_{1}^{S}=0$. The two sigma variables introduced for each statement are carefully linked to the $\beta$ coefficients representing the loop nesting structure: full tile distribution is easily detectable with $\beta_{0}^{R} \neq \beta_{0}^{S}$, making $\sigma_{1}^{R}=0$ and $\sigma_{1}^{S}=0$; the other two fusion scenarios require connecting $\sigma_{1}$ constraints to all scalar tile dimensions (i.e. the first $d$ scalar dimensions) while $\sigma_{2}$ variables need only compare $\beta_{2 d}^{R}$ and $\beta_{2 d}^{S}$. We note that these constraints must be created for every pair of statements.

Given the possible combination of values of sigmas, we then leverage the constraints in Equations 6-8 to model the three different memory quotas per dominating array reference, where $V_{f t d}$ is defined as the number of elements fitting into memory divided 
by the maximum number of dominating arrays references among all statements, $V_{p t r}$ is the number of memory elements (mem) split among all dominating arrays, and $V_{f t f}$ is equal to $V_{p t f}$ plus a slack obtained from redistributing $V_{p t f}$ among all the references to dominating arrays.

$$
\begin{array}{r}
\forall S, \forall F \in S: \\
\sum_{i=0}^{d-1} u \operatorname{sed}(F, i) \cdot \tau_{i}^{S} \leq V_{f t d} \wedge \\
\sum_{i=0}^{d-1} u \operatorname{sed}(F, i) \cdot \tau_{i}^{S} \leq V_{f t f}+m e m \cdot \sigma_{1}^{S} \wedge \\
\sum_{i=0}^{d-1} u \operatorname{sed}(F, i) \cdot \tau_{i}^{S} \leq V_{p t f}+m e m \cdot\left(\sigma_{1}^{S}+\sigma_{2}^{S}\right)
\end{array}
$$

Concrete Hardware Constraints. We adopt a minimal approach to modeling the hardware configuration of a GPU, and consider only three defining properties: i) the maximum work group (thread block) size; ii) the shared-memory capacity; iii) the presence of (true) vector units. We consider the first of these as the number of threads that will fit into a CUDA SM or a Compute Unit (CU) in an AMD GPU, with values of 2048 and 512, respectively. Thus, we require the tile sizes associated to the two outermost dimensions to be further constrained.

The maximum work group size, $W G S_{\max }$, is given as an input to our transformation engine, and is obtained by

$$
w g s=\log _{\text {tile_base }}\left(W G S_{\max }\right)
$$

We then introduce two new sets of variables per statement, grid size variables $g s_{0}^{S}$ and $g s_{1}^{S}$, and block size variables $b s_{0}^{S}$ and $b s_{1}^{S}$. We take advantage of some pre-defined values provided by PPCG, typically used as defaults for block size and grid size unless the user provides its own. We thus use this as upper bounds. In particular, PPCG uses $\{32,16\}$ for kernels able to extract 2D parallelism. The constraints used, for each program statement $S$, to limit and link the tile sizes to the previous variables are shown below:

$$
\begin{array}{r}
b s_{0}^{S} \leq\left\lceil\log _{\text {tile_base }}(32)\right\rceil \wedge b s_{1}^{S} \leq\left\lceil\log _{\text {tile_base }}(16)\right\rceil \\
\forall i \in\{0,1\}: g s_{i}^{S} \leq w g s \\
\tau_{0}^{S} \leq b s_{0}^{S} \wedge \tau_{1}^{S} \geq b s_{1}^{S} \\
\forall i \in\{0,1\}: g s_{i}^{S}+\tau_{i}^{S} \leq w g s \wedge g s_{i}^{S}+b s_{i}^{S} \leq w g s \\
\forall i \in\{0,1\}: g s_{i}^{S}-\tau_{i}^{S}+b s_{i}^{S} \leq \log _{\text {tile_base }}(P B S)
\end{array}
$$

Exploiting SIMD/SIMT in GPUs. Harnessing SIMD/SIMT parallelism is necessary to fully exploit the compute power of modern GPUs, some of which have true SIMD units (e.g. the AMD Radeon VII) and others that mimic it with SIMT capabilities (e.g. NVIDIA ones). We embed an objective to maximize the vectorization potential and contiguity access. In practice, this objective will favor loop transformations that will fully sink parallel loops with stride-1 access. We recall that our cross-compilation approach only provides tile sizes to be plugged into a GPU polyhedral compiler. As such, we don't control the actual loop transformations taking place in the target compiler. Nevertheless, the relevance of this objective is that all loop dimensions not being used for vectorization become candidates for exploiting the two-level parallelism required by GPU processors. We thus define this objective as:

$$
\begin{array}{r}
O_{\text {inner }}=\left\{\text { Lexmin } \sum_{\forall S} \sum_{\forall F \in S} \sum_{k \in F} \operatorname{cost}(F, k) . \theta_{4 d-1,2 k}^{S},\right. \\
\text { Lexmin } \left.\sum \delta_{4 d-1}^{R \rightarrow S}\right\}
\end{array}
$$

The above cost function is adapted from $[9,35]$, but considering the usage of a $4 d+1$ schedule representation. The first component of the objective minimizes the overall access stride, while the second one maximizes the potential for parallelism at the last linear schedule dimension (i.e. the innermost loop). For the stride access, $\operatorname{cost}(F, k)$ assumes the weights of 1,3 and 10 for stride- 1 , stride- 0 and stride-N (high stride) access, respectively as per [34, 35]. We also note that, we prefer to minimize first the stride access given the now common presence of L1 and L2 caches, which will be the fallback scenario if shared-memory is not possible.

Maximizing Outer Parallelism. Our approach to maximize outer parallelism consists of two objectives. First, we increase the separation among statements when they do not share any dominating reference (a small modification to the SIS objective of [35]) and set this as the leading objective $O_{\text {sis }}$; Second, we minimize the sum of dependences satisfied at each of the two outermost linear scheduling dimensions:

$$
O_{\text {outer }}=\operatorname{Lexmin} \sum_{\mathcal{D}_{R, S}} \delta_{1}^{\mathcal{D}_{R, S}}, \sum_{\mathcal{D}_{R, S}} \delta_{3}^{\mathcal{D}_{R, S}}
$$

The net effect of the above objective is to push dependence satisfaction to all other scheduling dimensions, including the outermost scalar dimension, and potentially leading to a higher number of fully distributed loop nests. We note, nonetheless, that this objective has a minor effect after maximizing the $O_{\text {tiling }}$ cost function, which pushes dependence satisfaction to non-tile linear schedule levels.

ILP Wrap-up. To conclude, the final objective function for our cross-compilation ILP is built as follows:

$$
\left\{O_{\text {sis }}, O_{\text {tiling }}, O_{\text {balance }}, O_{\text {outer }}, \forall S m e m f^{S}, O_{\text {inner }}\right\}
$$

The above ILP system is lexicographically solved, tile sizes are recovered and used as the exponents over the selected tile base. Lastly, as the true tile sizes (i.e. tile_base $e_{i}^{S}$ ) might result in floating point values somewhat far from an integer, we ceil these values and round them up the nearest multiple of 4 .

We make one final note, which is that our scheme can produce different tile sizes for each statement. As such, we proceed to a voting mechanism to select a single set of tile sizes to be fed to PPCG. If multiple non-repetitive tile sizes are produced, we simply choose the first one. 


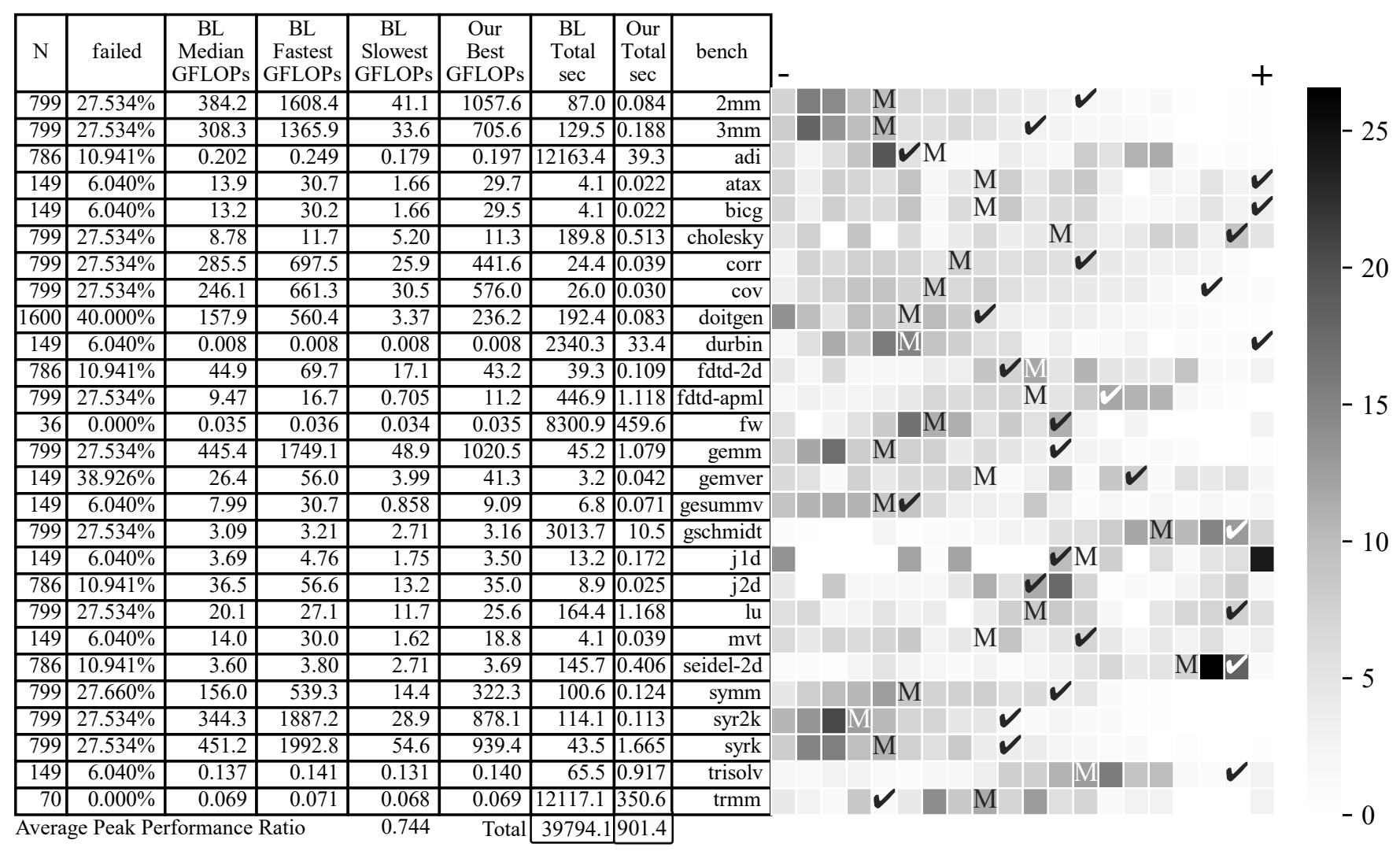

Figure 3: Table with performance statistics in GFlop/s (left) and heatmap for the performance of different benchmarks (right) on AMD GPU. Each row in the heatmap represents the percentage of tile configurations within each interval. $M$ indicates 'median' baseline performance and checkmark indicates our performance. (fastest is the rightmost)

\section{EXPERIMENTAL EVALUATION}

We implemented our tile size selection approach in the PoCC compiler, version $1.5^{2}$. The baseline polyhedral compiler used is PPCG 0.08.2, built with pet-0.11.2 and using isl-0.21-GMP. Our evaluation was performed on two different GPU platforms, an NVIDIA Tesla V100 (Volta GPU architecture) and an AMD Radeon VII. The technical specifications of both GPUs are shown in Table 1. The CUDA version used in our experiments is 9.1. In order to isolate the compute execution time among cuda kernel launches, the environment variable CUDA_LAUNCH_BLOCKING was set to 1 on the V100. For the AMD GPU, the ROCm v4.0.0 compiler infrastructure was used.

All experiments were conducted using the Polybench/C 3.2 benchmark suite, floating point double-precision (DP), and the default problem sizes of the LARGE_DATASET. A large tiled exploration space was generated for each benchmark and target architecture. The number of baseline configurations used for each benchmark varied in most cases between 149 and 799 distinct combinations of tile sizes, the exceptions being trmm (70 and 48),

\footnotetext{
${ }^{2}$ Prototype available at: https://github.com/mkongiv/polycross- comp-ics21.git
}

floyd-warshall (36 and 12) and doi tgen (up to 1600 configurations). For the former two, while tiling is legal, coarse-grained parallelization is not feasible, and requires extensive rewriting of the original code. The number of configurations tested varied primarily with the maximum loop depth of each benchmark. In addition, combinations of tile sizes were restricted by the maximum tile footprint of $32 \mathrm{~KB}$. However, as the final footprint and resource

Table 1: Technical Specifications for NVIDIA Tesla V100 GPU and AMD Radeon VII GPU

\begin{tabular}{|l|c|c|}
\hline & NVIDIA & AMD \\
\hline NVIDIA Tensor Cores & 640 & N/A \\
Total Cores & 5120 & 3840 \\
Stream Processors (SMs) & 80 & 60 \\
Peak Performance (DP) & $7.8 \mathrm{TFLOPs}$ & $3.46 \mathrm{TFLOPs}$ \\
GPU Memory & $16 \mathrm{~GB} \mathrm{HBM} 2$ & $16 \mathrm{~GB} \mathrm{HBM} 2$ \\
L2 Cache & $6 \mathrm{MB}$ & $4 \mathrm{MB}$ \\
Shared Memory & Up to $96 \mathrm{~KB}$ & $32 \mathrm{~KB}$ \\
Clock Frequency & $1530 \mathrm{MHz}$ & $1400 \mathrm{MHz}$ \\
Memory Bandwidth & $900 \mathrm{~GB} / \mathrm{s}$ & $1024 \mathrm{~GB} / \mathrm{s}$ \\
\hline
\end{tabular}




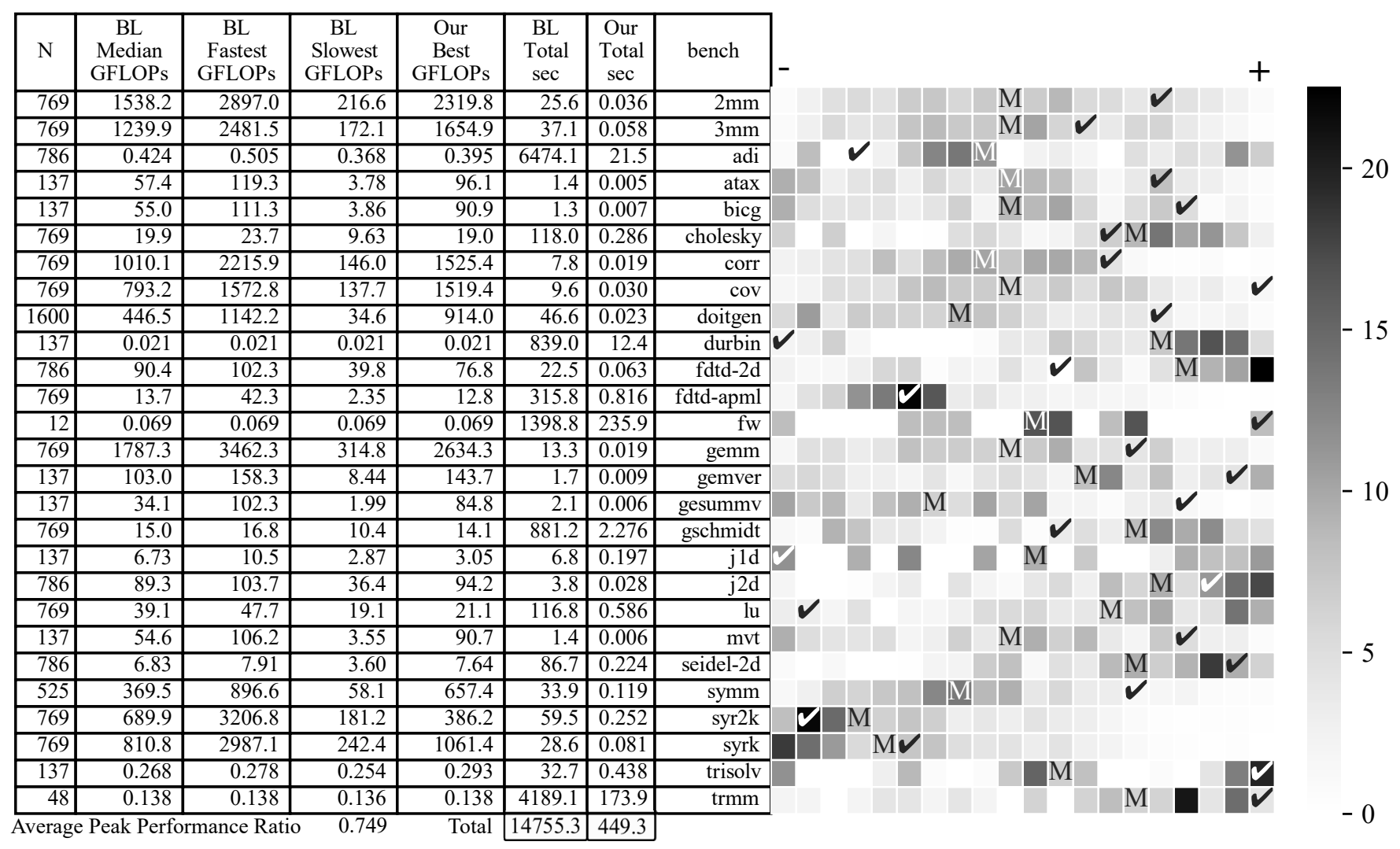

Figure 4: Table with performance statistics in GFlop/s (left) and heatmap for the performance of different benchmarks (right) on NVIDIA GPU. Each row in the heatmap represents the percentage of tile configurations within each interval. $M$ indicates 'median' baseline performance and checkmarks indicate our performance. (fastest is the rightmost)

usage of a kernel depends on the loop fusion structure chosen by PPCG, some fraction of the generated space failed to launch in the AMD GPU. Benchmarks symm, gramschmidt and cholesky were manually memory expanded to maximize tiling opportunities, similar to [70]. Each tile configuration was executed five times; The fastest and slowest runs for each configuration were excluded, and the average of the remaining ones used as the representative execution time for the tile configuration. For some benchmarks, we use a short-hand name instead of the full name such as: corr for correlation, cov for covariance, fw for floyd-warshall, gschmidt for gramschmidt, j1d for jacobi-1d-imper, and j2d for jacobi-2d-imper.

Figure 3 summarizes the results of our experiments on the AMD GPU. The left part of the figure shows a table with several metrics of interest for each benchmark: total number of generated tile configurations $(\mathrm{N})$, failed configurations during runtime as a percentage of the total generated configurations (failed), median performance for the baseline in GFlop/s (BL Median), the highest performance for the baseline in GFlop/s (BL Fastest), the lowest performance for the baseline in GFlop/s (BL Slowest), and our proposed technique best performance (higher of reuse and vector) in GFlop/s (Our Best), total execution time in seconds for the evaluated configurations for each benchmark (BL Total), and the total execution time in seconds for our reuse and vector variants (Our Total). At the bottom of the table, we report the Average Peak Performance Ratio, which we define as the ratio between our average best performance across all benchmarks (in GFlop/s), and the average best performance for the baseline configuration across all benchmarks (in GFlop/s). The right part of Figure 3 shows a heatmap for the baseline performance in GFlop/s. Each heatmap row represents 20 performance intervals of tile configurations, resulting from the best and worst performance in the joint evaluated space (PPCG and ours); The density of each interval indicates the percentage of the evaluated tile configurations that fall within the performance range for this interval. Performance intervals are ordered from the slowest (leftmost) to the fastest (rightmost). Checkmarks in a heatmap cell denote where our tile selection technique lies, in terms of performance, relative to the baseline intervals; Cells with an ' $M$ ' indicate the position of the median baseline performance. Figure 4 shows the corresponding statistics of our evaluation on the NVIDIA GPU, but ommiting the 'Failed' as almost no configurations failed on it (owing to its larger combined shared-memory and L1 cache), except for $13 \%$ of the evaluated doitgen configurations. 

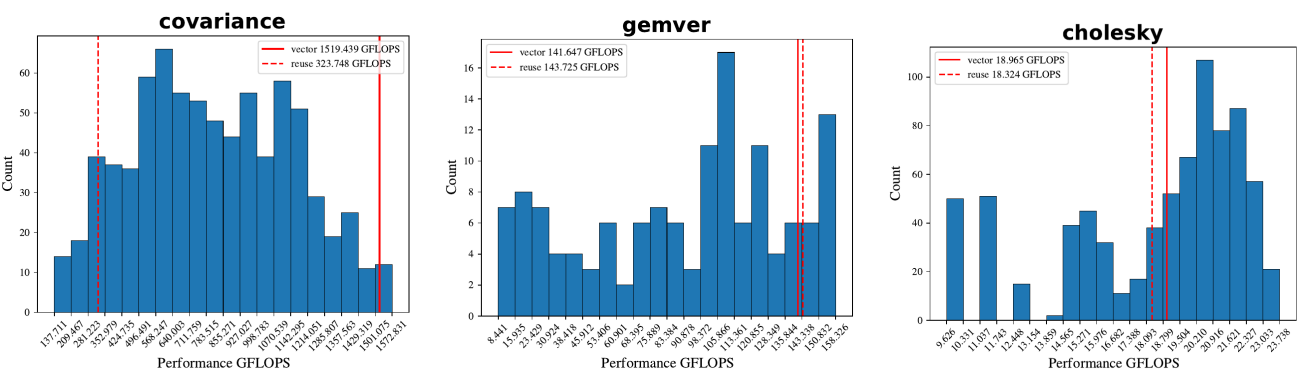

(a) NVIDIA Tesla V100.
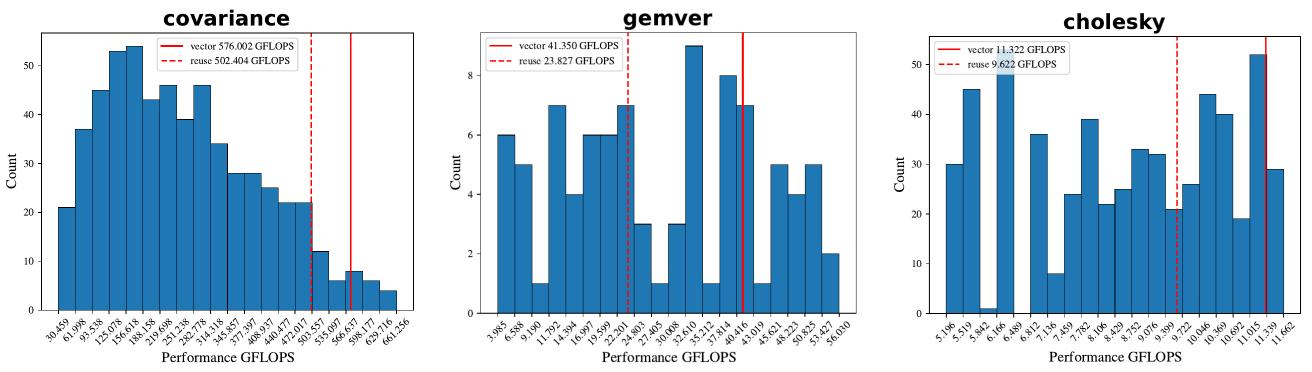

(b) AMD Radeon VII.
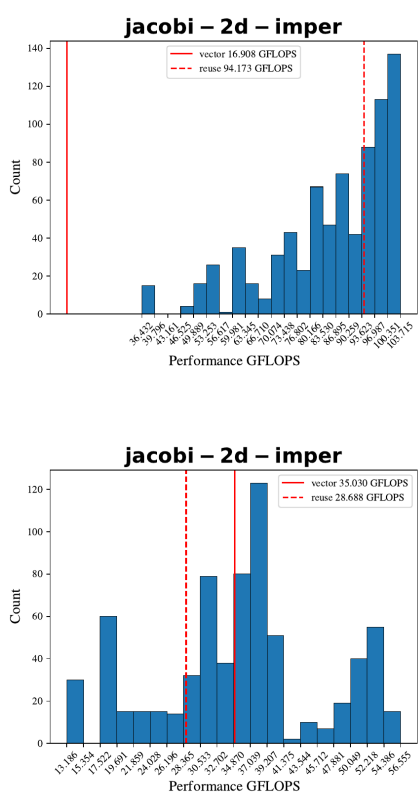

Figure 5: Histograms for the performance of covariance, gemver, cholesky, and jacobi-2d for both the evaluated baseline configurations and our technique on NVIDIA Tesla V100 (top) and AMD Radeon VII (bottom).

Inspecting the AMD results table and heatmap in Figure 3, we can observe that our technique achieves a higher performance than the median baseline performance in about $89 \%$ of the evaluated benchmarks, with up to $2.88 \times$ speedup over the median baseline performance. Our technique also achieves a higher performance than up to $99.3 \%$ of the evaluated configurations in some benchmarks, while averaging nearly $75 \%$ of the best empirically found tile configuration across all benchmarks.

On the NVIDIA GPU - Figure 4 - our technique achieves a peformance higher than the baseline's median in more than $70 \%$ of the evaluated benchmarks with up to $2.5 \times$ speedup over the median performance. Our tile selection bests up to $98.5 \%$ of the evaluated configurations in some benchmarks (e.g. covariance), and produces an almost identical average performance ratio, $74.9 \%$, of the best experimentally determined variant.

We can also notice the tremendous difference in exploration time between our approach and the baseline method, which exhausts a large space of tile possibilties (as shown in the bottom of the tables in Figures 3 and 4). For the AMD GPU, the baseline technique requires $44 \times$ more time than our technique while trying to find high performance parameters; On the NVIDIA GPU, our technique is $33 \times$ faster as well. This is an essential advantage of our technique as compared to evaluating hundreds (or even thousands) of tile configurations, that is, even if our technique does not guarantee the highest possible performance, it finds a strong performing configuration with a strong likelihood. Moreover, we believe our selection scheme to be particularly useful in scenarios where conducting a dedicated auto-tuning optimization phase for compute-intensive kernel is not possible.
Next, we focus our attention on the performance distribution of some interesting cases. Figure 5 shows the performance histograms for a subset of the Polybench suite: covariance, gemver, cholesky and jacobi-2d-imper. Horizontal axes show the performance in GFlop/s, while vertical axes show the count of configurations that lie within each performance interval. The top four histograms in Figure 5a correspond to the NVIDIA GPU, while the bottom four Figure $5 b$ - show the corresponding AMD histograms. Each plot also contains two vertical red lines marking the performance of our proposed technique when put in the baseline context; The solid line represents vector balance variant, while the dashed line indicates our reuse balance variant performance.

From the figures, we can make the following observations. First, in most cases our variants achieve a higher performance than most of the evaluated baseline tile configurations. Second, the vector balance variant normally achieves better performance than its reuse counterpart, yielding in some cases a non-negligible gap. As a reminder, this means that the tile size associated to the vector dimension has been preferred for maximization. The underlying reasons for the performance difference vary. For covariance, for example, the main computational kernel is a 3-dimensional triangular loop, with the vector dimension being conditioned by the outer iterator. Thus, maximizing the vector tile size, implicitly also decreases the (outer-) parallel granularity (i.e., produces more units of work to share). Conversely, the reuse balance variant yields higher granularity for coarse-grained parallelism (SM-level) and fewer intra-SM work. Moving to gemver, as a 2-dimensional kernel, favoring the tile size associated to the vector dimension equates, in principle, to reducing the parallelism granularity. This doesn't affect the AMD GPU in excess, owing to its true-vector units. However, 


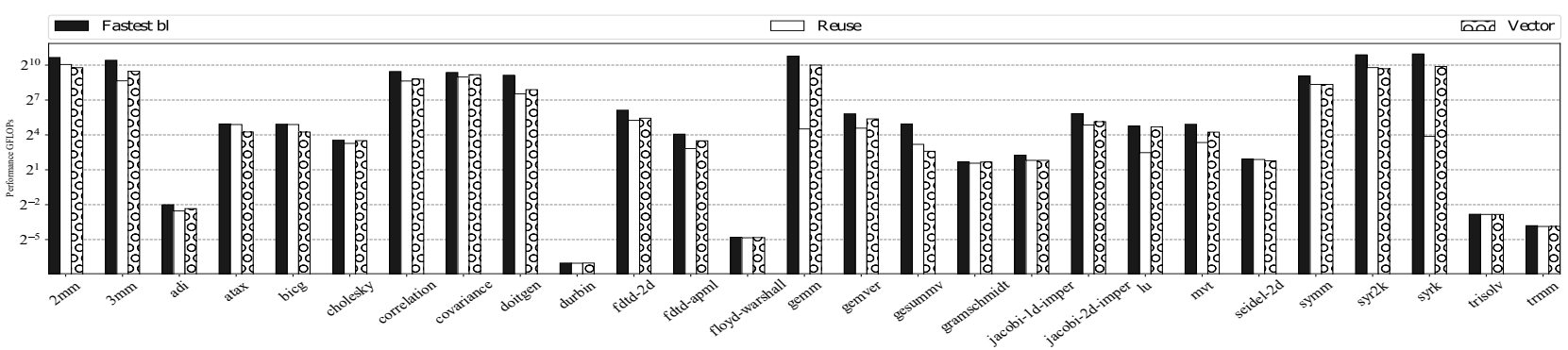

Figure 6: Clustered bar chart comparing the performance (GFlop/s) of our two variants: Reuse balance and Vector balance against the fastest baseline performance of the evaluated tile configurations on AMD Radeon VII GPU

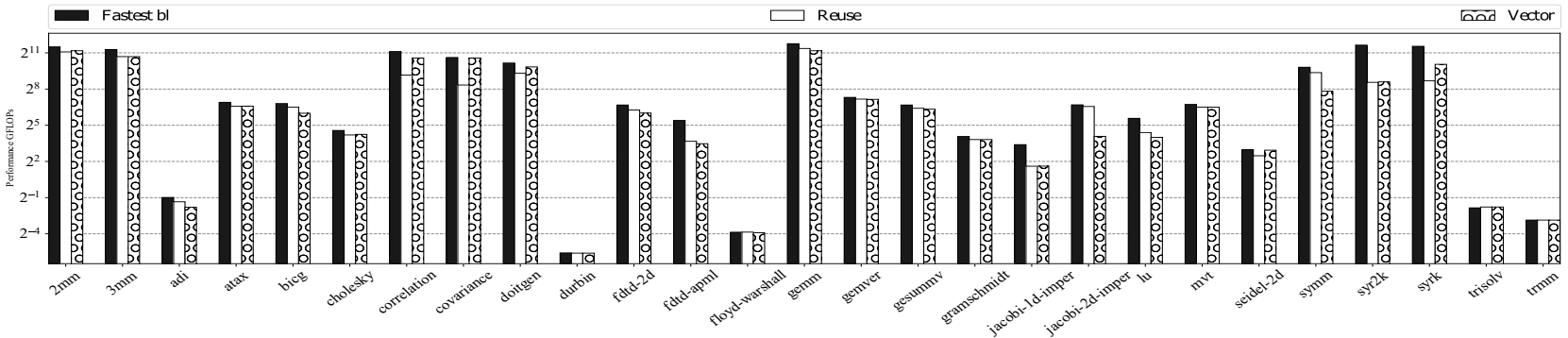

Figure 7: Clustered bar chart comparing the performance (GFlop/s) of our two variants: Reuse balance and Vector balance against the fastest baseline performance of the evaluated tile configurations on NVIDIA Tesla V100 GPU

on the V100, preferring larger tile sizes for the reuse balance variants significantly affects the third loopnest, which is dominated by a reference $A[j][i]$ (originally surrounded by the loop order $\langle i, j\rangle)$; In contrast to the previous two cases, the gap observed in the cholesky decomposition kernel is due to the outermost loop dimension not being tileable, which results in 4 CUDA/OpenCL kernels being repeatedly launched within a serial loop; This means that only two loop dimensions need to be considered. We also note that cholesky also exhibits a triangular loop, conditioning the vector dimension of the dominant kernel in a manner similar to covariance.

Lastly, for jacobi-2d, the reuse variant achieves a much better performance than the vector balance because the latter variant did not effectively tile the outermost space dimension (the $i$ dimension in the $\langle t, i, j\rangle$ loop order). This is owed to the "unbalanced" number of array references using $j$ as the vector index ( 6 in the first statement and 2 in the copy-back statement), while the $i$ iterator appears zero times; As a result, the i dimension is not tiled. Conversely, the reuse variant produced tile sizes $\{24,88,20\}$, even though the trip count for the time loop is set to 20 iterations. Similar effects are expected in computations with extreme unbalanced usage among loop iterators in the array vector dimensions.

To conclude our experimental evaluation, we compare the performance of our two variants, reuse balance and vector balance, against the best empirically found variant in the generated tiled space, on both the AMD Radeon VII GPU and the NVIDIA Tesla V100 GPU Fig. 6 and Fig. 7 - respectively. From both figures, it is noted that for all evaluated benchmarks our technique achieves a performance close to the fastest baseline configuration, while not requiring to resort to an exhaustive exploration of possible configurations. As can be observed, our tile size selection technique determines configurations that yield strong performance, usually comparable to the highest performance than can be achieved by generating hundreds of tile sizes combinations.

While highly-tuned libraries are expected to achieve a high fraction of the peak attainable machine performance, our technique stands out by quickly obtaining a practically high performance for general purpose applications without worrying much about peak attainable machine performance.

\section{RELATED WORK}

There is a large body of work in the area of loop tiling, including several polyhedral compiler frameworks such as PLuTo [8], Polly [21], PPCG [59], Polly-ACC [22] and PolyMage [43] (for image processing pipelines). As noted by Mullapudi et al. [43], even traversing the tile space configuration can lead to prohibitive costs due to potential combinatorial explosion in high-dimensional kernels. More recently, Jangda and Bondhugula [29] proposed a dynamic programming algorithm to effectively search the space of tile and fusion transformations. Our approach shares some similarities with [6] in that both leverage linear/affine constraints modeling the relations among schedule coefficients as exponents. However, the main motivation in [6] was to permit the modeling of negative coefficients (to permit, for instance, loop reversals) while avoiding the zero solution. In contrast, the techniques we propose leverage the legal space formulation while introducing several hardware notions that permit to find better tile configurations. 
Other works such as Flextended Tiles [64] improve on the traditional overlapped tiles [37] with the goal of reducing redundant computations. Previously, split tiling [20] and hexagonal tiling [19] were also used in multiple automated code generators, in particular, for iterative stencil computations, to enhance the parallelism. Due to the vastly different nature in domain-specific tiling approaches for GPUs, we defer to future work the adaptation of our technique to exploit non-rectangular tiling shapes. Yuki et al. [63] proposed the use of Neural Networks with the Scaled Conjugate Gradient method to derive tile sizes; Their approach limited the program representation to only 6 features, including prefetcher attributes per array reference. Li et al. [39] proposed a coordinated tiling and batching framework to improve General Matrix Multiplication (GEMM) on GPUs. It consists of two main phases: a tiling engine that carefully determines a tile size and assigns independent tiles to GPU thread blocks, and a batching engine to batch GEMM. Jangda and Guha [30] introduced an image processing pipeline execution technique that generates overlapped tiles fitting in a warp to reduce synchronization overhead.

Rawat et al. [51] introduced a domain-specific language called "STENCILGEN" to describe and generate optimized GPU code for stencil computations, leveraging multiple tiling techniques. AN5D [42] is another framework for automatic generation of optimized stencil GPU code, from generic $\mathrm{C}$ code. It also depends on different forms of temporal blocking. FlexTensor [66] is a framework that makes use of tiling and vectorization techniques to automatically explore and optimize tensor schedules on heterogeneous (CPU, GPU, and FPGA) systems. Wu et al. [62] proposed a runtime tiling framework for scratchpad memories to achieve a balance between bandwidth and space utilization. Rawat et al. [50] presented an automatic model-driven approach for code generation on GPUs guided by available resource information to adaptively implement fusion and time tiling. Lastly, Holewinski et al. [24] presented a GPU code generation technique for stencils to better exploit the use of shared memory while maximizing parallelism.

\section{CONCLUSIONS}

We have developed and presented a polyhedral cross-compilation technique to select suitable sets of tile sizes that permit us to achieve strong out-of-the-box performance. Our results on two GPGPU from different vendors, each using a different programming model, demonstrate the effectiveness of our approach, which achieves on average nearly $75 \%$ of the best experimentally found tile configuration. In addition, our approach also permits to significantly reduce the tuning time due to the minor need to test two variants. To the best of our knowledge this is the first polyhedral cross-compilation technique developed either for CPUs or GPUs.

\section{ACKNOWLEDGMENTS}

This research used resources of the Oak Ridge Leadership Computing Facility, which is a DOE Office of Science User Facility supported under Contract DE-AC05-00OR22725. This work used the Extreme Science and Engineering Discovery Environment (XSEDE), which is supported by National Science Foundation grant number ACI1548562. This research was supported in part by resources from
Stampede2, Texas Advanced Computer Center (TACC) XSEDE service provider, through allocation CCR190043.

\section{REFERENCES}

[1] Vinayaka Bandishti, Irshad Pananilath, and Uday Bondhugula. 2012. Tiling stencil computations to maximize parallelism. In SC'12: Proceedings of the International Conference on High Performance Computing, Networking, Storage and Analysis. IEEE, 1-11. https://doi.org/10.1109/SC.2012.107

[2] Muthu Manikandan Baskaran, Uday Bondhugula, Sriram Krishnamoorthy, J. Ramanujam, Atanas Rountev, and P. Sadayappan. 2008. A Compiler Framework for Optimization of Affine Loop Nests for Gpgpus. In Proceedings of the 22Nd Annual International Conference on Supercomputing (Island of Kos, Greece) (ICS '08). ACM, New York, NY, USA, 225-234. https://doi.org/10.1145/1375527.1375562

[3] Muthu Manikandan Baskaran, Albert Hartono, Sanket Tavarageri, Thomas Henretty, J. Ramanujam, and P. Sadayappan. 2010. Parameterized Tiling Revisited. In Proceedings of the 8th Annual IEEE/ACM International Symposium on Code Generation and Optimization (Toronto, Ontario, Canada) (CGO '10). Association for Computing Machinery, New York, NY, USA, 200-209. https: //doi.org/10.1145/1772954.1772983

[4] Cedric Bastoul. 2004. Code generation in the polyhedral model is easier than you think. In Proceedings. 13th International Conference on Parallel Architecture and Compilation Techniques, 2004. PACT 2004. 7-16. https://doi.org/10.1109/PACT. 2004.1342537

[5] Ian J. Bertolacci, Catherine Olschanowsky, Ben Harshbarger, Bradford L. Chamberlain, David G. Wonnacott, and Michelle Mills Strout. 2015. Parameterized Diamond Tiling for Stencil Computations with Chapel Parallel Iterators. In Proceedings of the 29th ACM on International Conference on Supercomputing (Newport Beach, California, USA) (ICS '15). Association for Computing Machinery, New York, NY, USA, 197-206. https://doi.org/10.1145/2751205.2751226

[6] Uday Bondhugula, Aravind Acharya, and Albert Cohen. 2016. The Pluto+ Algorithm: A Practical Approach for Parallelization and Locality Optimization of Affine Loop Nests. ACM Trans. Program. Lang. Syst. 38, 3, Article 12 (April 2016), 32 pages. https://doi.org/10.1145/2896389

[7] Uday Bondhugula, Vinayaka Bandishti, and Irshad Pananilath. 2017. Diamond Tiling: Tiling Techniques to Maximize Parallelism for Stencil Computations. IEEE Transactions on Parallel and Distributed Systems 28, 5 (May 2017), 1285-1298. https://doi.org/10.1109/TPDS.2016.2615094

[8] Uday Bondhugula, Albert Hartono, J. Ramanujam, and P. Sadayappan. 2008. A Practical Automatic Polyhedral Parallelizer and Locality Optimizer. In Proceedings of the 29th ACM SIGPLAN Conference on Programming Language Design and Implementation (Tucson, AZ, USA) (PLDI '08). ACM, New York, NY, USA, 101113. https://doi.org/10.1145/1375581.1375595

[9] Lorenzo Chelini, Tobias Gysi, Tobias Grosser, Martin Kong, and Henk Corporaal. 2020. Automatic Generation of Multi-Objective Polyhedral Compiler Transformations. In Proceedings of the International Conference on Parallel Architectures and Compilation Techniques (Atlanta, Georgia, USA) (PACT 2020). ACM, New York, NY, USA.

[10] Chun Chen, Jacqueline Chame, and Mary Hall. 2008. CHiLL: A framework for composing high-level loop transformations. Technical Report. USC Computer Science.

[11] Stephanie Coleman and Kathryn S. McKinley. 1995. Tile Size Selection Using Cache Organization and Data Layout. In Proceedings of the ACM SIGPLAN 1995 Conference on Programming Language Design and Implementation (La Jolla, California, USA) (PLDI '95). Association for Computing Machinery, New York, NY, USA, 279-290. https://doi.org/10.1145/207110.207162

[12] Roshan Dathathri, Ravi Teja Mullapudi, and Uday Bondhugula. 2016. Compiling Affine Loop Nests for a Dynamic Scheduling Runtime on Shared and Distributed Memory. ACM Trans. Parallel Comput. 3, 2, Article 12 (July 2016), 28 pages. https://doi.org/10.1145/2948975

[13] Roshan Dathathri, Chandan Reddy, Thejas Ramashekar, and Uday Bondhugula. 2013. Generating efficient data movement code for heterogeneous architectures with distributed-memory. In Proceedings of the 22nd International Conference on Parallel Architectures and Compilation Techniques. 375-386. https://doi.org/10. 1109/PACT.2013.6618833

[14] Paul Feautrier. 1988. Parametric integer programming. RAIRO-Operations Research 22, 3 (1988), 243-268.

[15] Paul Feautrier. 1991. Dataflow analysis of array and scalar references. International Journal of Parallel Programming 20, 1 (01 Feb 1991), 23-53. https: //doi.org/10.1007/BF01407931

[16] Matteo Frigo and Volker Strumpen. 2005. Cache Oblivious Stencil Computations. In Proceedings of the 19th Annual International Conference on Supercomputing (Cambridge, Massachusetts) (ICS '05). Association for Computing Machinery, New York, NY, USA, 361-366. https://doi.org/10.1145/1088149.1088197

[17] Matteo Frigo and Volker Strumpen. 2009. The cache complexity of multithreaded cache oblivious algorithms. Theory of Computing Systems 45, 2 (2009), 203-233. 
[18] Sylvain Girbal, Nicolas Vasilache, Cédric Bastoul, Albert Cohen, David Parello, Marc Sigler, and Olivier Temam. 2006. Semi-automatic Composition of Loop Transformations for Deep Parallelism and Memory Hierarchies. Int. 7. Parallel Program. 34, 3 (June 2006), 261-317. https://doi.org/10.1007/s10766-006-0012-3

[19] Tobias Grosser, Albert Cohen, Justin Holewinski, P. Sadayappan, and Sven Verdoolaege. 2014. Hybrid Hexagonal/Classical Tiling for GPUs. In Proceedings of Annual IEEE/ACM International Symposium on Code Generation and Optimization (Orlando, FL, USA) (CGO '14). ACM, New York, NY, USA, Article 66, 10 pages. https://doi.org/10.1145/2544137.2544160

[20] Tobias Grosser, Albert Cohen, Paul H. J. Kelly, J. Ramanujam, P. Sadayappan, and Sven Verdoolaege. 2013. Split Tiling for GPUs: Automatic Parallelization Using Trapezoidal Tiles. In Proceedings of the 6th Workshop on General Purpose Processor Using Graphics Processing Units (Houston, Texas, USA) (GPGPU-6). Association for Computing Machinery, New York, NY, USA, 24-31. https://doi.org/10.1145/ 2458523.2458526

[21] Tobias Grosser, Armin Groesslinger, and Christian Lengauer. 2012 Polly-performing polyhedral optimizations on a low-level intermediate representation. Parallel Processing Letters 22, 04 (2012), 1250010.

[22] Tobias Grosser and Torsten Hoefler. 2016. Polly-ACC Transparent Compilation to Heterogeneous Hardware. In Proceedings of the 2016 International Conference on Supercomputing (Istanbul, Turkey) (ICS '16). ACM, New York, NY, USA, Article 1, 13 pages. https://doi.org/10.1145/2925426.2926286

[23] Tobias Grosser, Sven Verdoolaege, and Albert Cohen. 2015. Polyhedral AST Generation Is More Than Scanning Polyhedra. ACM Trans. Program. Lang. Syst. 37, 4, Article 12 (July 2015), 50 pages. https://doi.org/10.1145/2743016

[24] Justin Holewinski, Louis-Noël Pouchet, and P. Sadayappan. 2012. HighPerformance Code Generation for Stencil Computations on GPU Architectures. In Proceedings of the 26th ACM International Conference on Supercomputing (San Servolo Island, Venice, Italy) (ICS '12). Association for Computing Machinery, New York, NY, USA, 311-320. https://doi.org/10.1145/2304576.2304619

[25] Changwan Hong, Aravind Sukumaran-Rajam, Israt Nisa, Kunal Singh, and P. Sadayappan. 2019. Adaptive Sparse Tiling for Sparse Matrix Multiplication. In Proceedings of the 24th Symposium on Principles and Practice of Parallel Programming (Washington, District of Columbia) (PPoPP '19). Association for Computing Machinery, New York, NY, USA, 300-314. https://doi.org/10.1145/3293883.3295712

[26] Guillaume Iooss, Sanjay Rajopadhye, Christophe Alias, and Yun Zou. 2015. Monoparametric Tiling is a Polyhedral Transformation. Research Report RR-8802. INRIA Grenoble - Rhône-Alpes ; CNRS. 40 pages. https://hal.inria.fr/hal-01219452

[27] François Irigoin and Rémi Triolet. 1988. Supernode Partitioning. In Proceedings of the 15th ACM SIGPLAN-SIGACT Symposium on Principles of Programming Languages (San Diego, California, USA) (POPL '88). Association for Computing Machinery, New York, NY, USA, 319-329. https://doi.org/10.1145/73560.73588

[28] Alexandre Isoard. 2014. Data-Reuse Optimizations for Pipelined Tiling with Parametric Tile Sizes. In Proceedings of the 23rd International Conference on Paralle Architectures and Compilation (Edmonton, AB, Canada) (PACT '14). Association for Computing Machinery, New York, NY, USA, 509-510. https://doi.org/10 $1145 / 2628071.2671425$

[29] Abhinav Jangda and Uday Bondhugula. 2018. An Effective Fusion and Tile Size Model for Optimizing Image Processing Pipelines. In Proceedings of the $23 \mathrm{rd} \mathrm{ACM}$ SIGPLAN Symposium on Principles and Practice of Parallel Programming (Vienna, Austria) (PPoPP '18). ACM, New York, NY, USA, 261-275. https://doi.org/10. $1145 / 3178487.3178507$

[30] Abhinav Jangda and Arjun Guha. 2020. Model-Based Warp Overlapped Tiling for Image Processing Programs on GPUs. In Proceedings of the ACM International Conference on Parallel Architectures and Compilation Techniques (Virtual Event, GA, USA) (PACT '20). Association for Computing Machinery, New York, NY, USA, 317-328. https://doi.org/10.1145/3410463.3414649

[31] DaeGon Kim, Lakshminarayanan Renganarayanan, Dave Rostron, Sanjay Rajopadhye, and Michelle Mills Strout. 2007. Multi-Level Tiling: $M$ for the Price of One. In Proceedings of the 2007 ACM/IEEE Conference on Supercomputing (Reno, Nevada) (SC '07). Association for Computing Machinery, New York, NY, USA, Article 51, 12 pages. https://doi.org/10.1145/1362622.1362691

[32] Toru Kisuki, Peter MW Knijnenburg, and Michael FP O’Boyle. 2000. Combined selection of tile sizes and unroll factors using iterative compilation. In Proceedings 2000 International Conference on Parallel Architectures and Compilation Techniques (Cat. No.PR00622). IEEE, 237-246. https://doi.org/10.1109/PACT.2000.888348

[33] Martin Kong, Antoniu Pop, Louis-Noël Pouchet, R. Govindarajan, Albert Cohen, and P. Sadayappan. 2015. Compiler/Runtime Framework for Dynamic Dataflow Parallelization of Tiled Programs. ACM Trans. Archit. Code Optim. 11, 4, Article 61 (Jan. 2015), 30 pages. https://doi.org/10.1145/2687652

[34] Martin Kong and Louis-Noël Pouchet. 2018. A Performance Vocabulary for Affine Loop Transformations. CoRR abs/1811.06043 (2018). arXiv:1811.06043 http://arxiv.org/abs/1811.06043

[35] Martin Kong and Louis-Noël Pouchet. 2019. Model-driven Transformations for Multi- and Many-core CPUs. In Proceedings of the 40th ACM SIGPLAN Conference on Programming Language Design and Implementation (Phoenix, AZ, USA) (PLDI 2019). ACM, New York, NY, USA, 469-484. https://doi.org/10.1145/3314221. 3314653
[36] Martin Kong, Louis-Noël Pouchet, P. Sadayappan, and Vivek Sarkar. 2016. PIPES: A Language and Compiler for Task-Based Programming on Distributed-Memory Clusters. In SC '16: Proceedings of the International Conference for High Performance Computing, Networking, Storage and Analysis. 456-467. https://doi.org/10.1109/ SC.2016.38

[37] Sriram Krishnamoorthy, Muthu Baskaran, Uday Bondhugula, J. Ramanujam, Atanas Rountev, and P Sadayappan. 2007. Effective Automatic Parallelization of Stencil Computations. In Proceedings of the 28th ACM SIGPLAN Conference on Programming Language Design and Implementation (San Diego, California, USA) (PLDI '07). ACM, New York, NY, USA, 235-244. https://doi.org/10.1145/1250734. 1250761

[38] Allen Leung, Nicolas Vasilache, Benoît Meister, Muthu Baskaran, David Wohlford, Cédric Bastoul, and Richard Lethin. 2010. A Mapping Path for multi-GPGPU Accelerated Computers from a Portable High Level Programming Abstraction. In Proceedings of the 3rd Workshop on General-Purpose Computation on Graphics Processing Units (Pittsburgh, Pennsylvania, USA) (GPGPU-3). ACM, New York, NY, USA, 51-61. https://doi.org/10.1145/1735688.1735698

[39] Xiuhong Li, Yun Liang, Shengen Yan, Liancheng Jia, and Yinghan Li. 2019. A Coordinated Tiling and Batching Framework for Efficient GEMM on GPUs. In Proceedings of the 24th Symposium on Principles and Practice of Parallel Programming (Washington, District of Columbia) (PPoPP '19). Association for Computing Machinery, New York, NY, USA, 229-241. https://doi.org/10.1145/3293883.3295734

[40] Amy W. Lim, Gerald I. Cheong, and Monica S. Lam. 1999. An affine partitioning algorithm to maximize parallelism and minimize communication. In Proceedings of the 13th international conference on Supercomputing, ICS 1999, Rhodes, Greece, fune 20-25, 1999. 228-237. https://doi.org/10.1145/305138.305197

[41] Jianqiao Liu, Nikhil Hegde, and Milind Kulkarni. 2016. Hybrid CPU-GPU Scheduling and Execution of Tree Traversals. In Proceedings of the 21st ACM SIGPLAN Symposium on Principles and Practice of Parallel Programming (Barcelona, Spain) (PPoPP '16). ACM, New York, NY, USA, Article 41, 2 pages. https: //doi.org/10.1145/2851141.2851174

[42] Kazuaki Matsumura, Hamid Reza Zohouri, Mohamed Wahib, Toshio Endo, and Satoshi Matsuoka. 2020. AN5D: Automated Stencil Framework for High-Degree Temporal Blocking on GPUs. In Proceedings of the 18th ACM/IEEE International Symposium on Code Generation and Optimization (San Diego, CA, USA) (CGO 2020). Association for Computing Machinery, New York, NY, USA, 199-211. https://doi.org/10.1145/3368826.3377904

[43] Ravi Teja Mullapudi, Vinay Vasista, and Uday Bondhugula. 2015. PolyMage: Automatic Optimization for Image Processing Pipelines. In Proceedings of the Twentieth International Conference on Architectural Support for Programming Languages and Operating Systems (Istanbul, Turkey) (ASPLOS '15). ACM, New York, NY, USA, 429-443. https://doi.org/10.1145/2694344.2694364

[44] Daniel Orozco and Guang Gao. 2009. Diamond tiling: A tiling framework for time-iterated scientific applications. CAPSL Technical Memo 091, Tech. Rep. (2009).

[45] PoCC. 2018. The Polyhedral Compiler Collection. https://sourceforge.net/ projects/pocc/ Online; v1.5; accessed on August 2020.

[46] Louis-Noël Pouchet. 2012. Polybench: The polyhedral benchmark suite. URL: http://polybench.sf.net (2012).

[47] Louis-Noël Pouchet, Uday Bondhugula, Cédric Bastoul, Albert Cohen, J. Ramanujam, P. Sadayappan, and Nicolas Vasilache. 2011. Loop Transformations: Convexity, Pruning and Optimization. In Proceedings of the 38th Annual ACM SIGPLAN-SIGACT Symposium on Principles of Programming Languages (Austin, Texas, USA) (POPL '11). Association for Computing Machinery, New York, NY, USA, 549-562. https://doi.org/10.1145/1926385.1926449

[48] Jagannathan Ramanujam and Ponnuswamy Sadayappan. 1992. Tiling multidimensional iteration spaces for multicomputers. F. Parallel and Distrib. Comput. 16, 2 (1992), 108-120.

[49] Fabrice Rastello and Thierry Dauxois. 2002. Efficient Tiling for an ODE Discrete Integration Program: Redundant Tasks Instead of Trapezoidal Shaped-Tiles. In ipdps.

[50] Prashant Singh Rawat, Changwan Hong, Mahesh Ravishankar, Vinod Grover, Louis-Noel Pouchet, Atanas Rountev, and P. Sadayappan. 2016. Resource Conscious Reuse-Driven Tiling for GPUs. In Proceedings of the 2016 International Conference on Parallel Architectures and Compilation (Haifa, Israel) (PACT '16). Association for Computing Machinery, New York, NY, USA, 99-111. https: //doi.org/10.1145/2967938.2967967

[51] Prashant Singh Rawat, Miheer Vaidya, Aravind Sukumaran-Rajam, Mahesh Ravishankar, Vinod Grover, Atanas Rountev, Louis-Noël Pouchet, and P. Sadayappan. 2018. Domain-Specific Optimization and Generation of High-Performance GPU Code for Stencil Computations. Proc. IEEE 106, 11 (2018), 1902-1920. https://doi.org/10.1109/JPROC.2018.2862896

[52] Michelle Mills Strout, Larry Carter, Jeanne Ferrante, and Barbara Kreaseck. 2004. Sparse tiling for stationary iterative methods. The International fournal of High Performance Computing Applications 18, 1 (2004), 95-113.

[53] Michelle Mills Strout, Fabio Luporini, Christopher D Krieger, Carlo Bertolli, Gheorghe-Teodor Bercea, Catherine Olschanowsky, J Ramanujam, and Paul HJ Kelly. 2014. Generalizing run-time tiling with the loop chain abstraction. In 2014 IEEE 28th International Parallel and Distributed Processing Symposium. IEEE, 
$1136-1145$.

[54] Kirshanthan Sundararajah and Milind Kulkarni. 2019. Composable, Sound Transformations of Nested Recursion and Loops. In Proceedings of the 40th ACM SIGPLAN Conference on Programming Language Design and Implementation (Phoenix, AZ, USA) (PLDI 2019). ACM, New York, NY, USA, 902-917. https://doi.org/10.1145/3314221.3314592

[55] Sanket Tavarageri, Albert Hartono, Muthu Baskaran, Louis-Noël Pouchet, J Ramanujam, and P Sadayappan. 2010. Parametric tiling of affine loop nests. In Proc. 15th Workshop on Compilers for Parallel Computers. Vienna, Austria.

[56] Ananta Tiwari, Chun Chen, Jacqueline Chame, Mary Hall, and Jeffrey K. Hollingsworth. 2009. A Scalable Auto-tuning Framework for Compiler Optimization. In Proceedings of the 2009 IEEE International Symposium on Parallel\&Distributed Processing (IPDPS '09). IEEE Computer Society, Washington, DC, USA, 1-12. https://doi.org/10.1109/IPDPS.2009.5161054

[57] Nicolas Vasilache. 2007. Scalable Program Optimization Techniques in the Polyhedra Model. Ph.D. Dissertation. University of Paris-Sud 11.

[58] Anand Venkat, Manu Shantharam, Mary Hall, and Michelle Mills Strout. 2014 Non-Affine Extensions to Polyhedral Code Generation. In Proceedings of Annual IEEE/ACM International Symposium on Code Generation and Optimization (Orlando, FL, USA) (CGO '14). Association for Computing Machinery, New York, NY, USA, 185-194. https://doi.org/10.1145/2581122.2544141

[59] Sven Verdoolaege, Juan Carlos Juega, Albert Cohen, José Ignacio Gómez, Christian Tenllado, and Francky Catthoor. 2013. Polyhedral Parallel Code Generation for CUDA. ACM Trans. Archit. Code Optim. 9, 4, Article 54 (Jan. 2013), 23 pages. https://doi.org/10.1145/2400682.2400713

[60] Michael Wolfe. 1989. More Iteration Space Tiling. In Proceedings of the 1989 ACM/IEEE Conference on Supercomputing (Reno, Nevada, USA) (Supercomputing '89). Association for Computing Machinery, New York, NY, USA, 655-664. https: //doi.org/10.1145/76263.76337

[61] David G Wonnacott and Michelle Mills Strout. 2013. On the scalability of loop tiling techniques. IMPACT 20133 (2013).

[62] Mingchuan Wu, Ying Liu, Huimin Cui, Qingfu Wei, Quanfeng Li, Limin Li, Fang Lv, Jingling Xue, and Xiaobing Feng. 2020. Bandwidth-Aware Loop Tiling for DMA-Supported Scratchpad Memory. In Proceedings of the ACM International Conference on Parallel Architectures and Compilation Techniques (Virtual Event, GA, USA) (PACT '20). Association for Computing Machinery, New York, NY, USA 97-109. https://doi.org/10.1145/3410463.3414637

[63] Tomofumi Yuki, Lakshminarayanan Renganarayanan, Sanjay Rajopadhye, Charles Anderson, Alexandre E. Eichenberger, and Kevin O'Brien. 2010. Automatic Creation of Tile Size Selection Models. In Proceedings of the 8th Annual IEEE/ACM International Symposium on Code Generation and Optimization (Toronto, Ontario, Canada) (CGO '10). Association for Computing Machinery, New York, NY, USA, 190-199. https://doi.org/10.1145/1772954.1772982

[64] Jie Zhao and Albert Cohen. 2019. Flextended Tiles: A Flexible Extension of Overlapped Tiles for Polyhedral Compilation. ACM Trans. Archit. Code Optim. 16, 4, Article 47 (Dec. 2019), 25 pages. https://doi.org/10.1145/3369382

[65] Jiacheng Zhao, Huimin Cui, Yalin Zhang, Jingling Xue, and Xiaobing Feng. 2018 Revisiting Loop Tiling for Datacenters: Live and Let Live. In Proceedings of the 2018 International Conference on Supercomputing (Beijing, China) (ICS '18) Association for Computing Machinery, New York, NY, USA, 328-340. https //doi.org/10.1145/3205289.3205306

[66] Size Zheng, Yun Liang, Shuo Wang, Renze Chen, and Kaiwen Sheng. 2020. FlexTensor: An Automatic Schedule Exploration and Optimization Framework for Tensor Computation on Heterogeneous System. In Proceedings of the Twenty-Fifth International Conference on Architectural Support for Programming Languages and Operating Systems (Lausanne, Switzerland) (ASPLOS '20). Association for Computing Machinery, New York, NY, USA, 859-873. https://doi.org/10.1145/ 3373376.3378508

[67] Xing Zhou, Jean-Pierre Giacalone, María Jesús Garzarán, Robert H Kuhn, Yang $\mathrm{Ni}$, and David Padua. 2012. Hierarchical overlapped tiling. In Proceedings of the Tenth International Symposium on Code Generation and Optimization. 207-218.

[68] Hans Zima, Mary Hall, Chun Chen, and Jaqueline Chame. 2009. Model-guided Autotuning of High-productivity Languages for Petascale Computing. In Proceedings of the 18th ACM International Symposium on High Performance Distributed Computing (Garching, Germany) (HPDC '09). ACM, New York, NY, USA, 151-166. https://doi.org/10.1145/1551609.1551611

[69] Oleksandr Zinenko, Sven Verdoolaege, Chandan Reddy, Jun Shirako, Tobias Grosser, Vivek Sarkar, and Albert Cohen. 2017. Unified Polyhedral Modeling of Temporal and Spatial Locality. Research Report RR-9110. Inria Paris. 41 pages. https://hal.inria.fr/hal-01628798

[70] Oleksandr Zinenko, Sven Verdoolaege, Chandan Reddy, Jun Shirako, Tobias Grosser, Vivek Sarkar, and Albert Cohen. 2018. Modeling the Conflicting Demands of Parallelism and Temporal/Spatial Locality in Affine Scheduling. In Proceedings of the 27th International Conference on Compiler Construction (Vienna, Austria) (CC 2018). ACM, New York, NY, USA, 3-13. https://doi.org/10.1145/ 3178372.3179507 